\title{
Mapeamento de Estressores no Trabalho de Expedicionários do Programa Antártico Brasileiro (PROANTAR)
}

\author{
Paola Barros-Delben* (D), Gustavo Klauberg Pereira, Hiago Murilo de Melo (D), \\ André Luiz Thieme, \& Roberto Moraes Cruz (D) \\ Universidade Federal de Santa Catarina, Florianópolis, SC, Brasil
}

\begin{abstract}
RESUMO - O programa Antártico Brasileiro (PROANTAR) realiza expedições à Antártica, onde militares e civis são expostos a estressores. A presente pesquisa mapeou os estressores ambientais, ocupacionais e interpessoais percebidos por participantes do PROANTAR. Uma amostra de 38 pessoas, separadas em dois grupos, foi avaliada no início e final de uma expedição. Os resultados obtidos por meio de questionários e entrevistas indicaram prevalência de estressores ambientais $(60,71 \%)$, ocupacionais $(23,80 \%)$ e interpessoais $(15,47 \%)$ no início, e de estressores interpessoais $(55,97 \%)$, ambientais $(32,08 \%)$ e ocupacionais $(11,94 \%)$ ao final. Os resultados sugerem que a convivência forçada gera a percepção de estressores interpessoais se sobrepondo aos ambientais. Fenômenos psicológicos deveriam ser considerados no planejamento de futuras expedições, pois estão relacionados à saúde e desempenho das atividades.
\end{abstract}

PALAVRAS-CHAVE: estresse, Antártica, psicologia organizacional e do trabalho

\section{Mapping of Stressors in the Work of Expeditionary Brazilian Antarctic Program (PROANTAR)}

\begin{abstract}
The Brazilian Antarctic Program (PROANTAR) conducts expeditions to Antarctica, where military and civilian are exposed to stressors. The present research mapped the environmental, occupational and interpersonal stressors perceived by PROANTAR participants. A sample of 38 people, separated into two groups, was evaluated at the beginning and end of an expedition. The results obtained through questionnaires and interviews indicated the prevalence of environmental (60.71\%), occupational (23.80\%) and interpersonal (15.47\%) stressors at the beginning, and interpersonal (55.97\%), environmental (32.08\%) and occupational (11.94\%) stressors at the end. The results suggest that the forced coexistence generates the perception of interpersonal stressors overlapping environmental ones. Psychological phenomena should be considered in the planning of future expeditions, as they are related to health and performance of activities.
\end{abstract}

KEYWORDS: stress, Antarctic, organizational and work psychology

O continente antártico é considerado um ambiente isolado, confinado e extremo (ICE), assim como desertos, montanhas e estações espaciais (Cobra, 2008; Leveton, 2014; Schmidt, Landon, \& Patterson, 2015). Ambientes ICE apresentam potenciais riscos de gerar impactos negativos em seres humanos, seja pelas características físicas do local ou pelos propósitos que levam homens e mulheres a trabalhar no continente austral.

Produzir conhecimento científico sobre fenômenos psicológicos em ambientes ICE possibilita o desenvolvimento de meios mais eficazes e eficientes de preparação e enfrentamento das situações. Alterações cognitivas, emocionais e comportamentais podem ocorrer nesses ambientes, considerando ainda as variações geradas pelas peculiaridades de cada grupo e cada objetivo de expedição (Corbett, Middleton, \& Arendt, 2012; Nicolas, Sandal, Weiss, \& Yusupova, 2013; Suedfeld, 2012; Weymouth \& Steel, 2013).

As pesquisas científicas na Antártica são realizadas por países membros consultivos do Tratado da Antártica, de 1975

Apoio: $\mathrm{CNPq}$

*E-mail: p.barros.delben@gmail.com

n Submetido em: 23/07/2016; Revisado: 07/06/2017; Aceito: 25/05/2018. 
(Baldrighi, 2016). O Brasil está incluso desde 1982, com o Programa Antártico Brasileiro (PROANTAR), coordenado pela Secretaria Interministerial para Recursos do Mar (SECIRM), subordinada ao comando da Marinha (Cobra, 2009; Jesus \& Souza, 2007). A SECIRM é responsável pelas atividades de logística, seleção e treinamento dos expedicionários e, também, pela manutenção da Estação Antártica Comandante Ferraz (EACF) e dos acampamentos, fornecendo suprimentos e deslocando pessoal. A Força Aérea Brasileira auxilia o PROANTAR, realizando voos programados e emergenciais (Freitas, 2012; Madureira Junior, 2014).

Os principais estudos científicos na Antártica estão relacionados ao meio ambiente, biologia e meteorologia. As pesquisas internacionais revelam interesse crescente na compreensão do papel das interações humanas no ambiente antártico, como demonstra o levantamento feito por Silva, Cabral e Zimmer (2014). Desde o ano polar de 1955 a 1957, a Psicologia, a Medicina e a Fisiologia se estabeleceram na terceira área polar de investigação no contexto (Gandra, 2013; Halsey \& Stroud, 2012). Nomes importantes destacam-se como precursores da Psicologia na Antártica e no Ártico, como Eric Gunderson, dos EUA, Jean Rivolier, da França, e Tony Taylor, da Nova Zelândia (Bishop, Kobrick, Battler, \& Binsted, 2010; Guly, 2012).

Internacionalmente, os principais estudos da Psicologia na Antártica dizem respeito a estresse, coping e estados de humor (Burns \& Sullivan, 2000; Zimmer, Cabral, Borges, Coco \& Hameister, 2013). Há, também, estudos sobre as alterações sazonais na condição física e mental dos expedicionários nessas regiões (Steinach et al., 2015), ou seja, alterações típicas que acometem as pessoas durante as estações do ano mais extremadas em altas latitudes (Broughton, 2016; Donoghue, 2016; Feuerecker et al., 2014; Flynn-Evans, Gregory, Arsintescu \& Whitmire, 2016; Landon, Vessey, \& Barrett, 2015; Premkumar, Sable, Dhanwal, \& Dewan, 2013).

A literatura psicológica nacional possui uma produção incipiente sobre a Psicologia na Antártica, já que somente uma autora (Cobra, 2008, 2009) publicou sobre a definição da Psicologia Polar e interação de grupos no contexto da Antártica, sem inserção in loco. Posteriormente, Zimmer, Silva e colaboradores (Silva et al., 2014; Zimmer et al., 2013) tiveram trabalhos publicados de revisão sistemática a respeito de aspectos psicológicos na Antártica e um levantamento das produções nacionais e internacionais sobre pesquisas nas áreas humanas. As demais publicações compreendem estudos apresentados em eventos científicos (Barros-Delben, Pereira, \& Cruz, 2017; Barros-Delben, Sombrio, Melo, Pereira, \& Cruz, 2015; Melo, Barros-
Delben, Sombrio, \& Cruz, 2015 Pereira, Barros-Delben, Melo, Mendonça, \& Cruz, 2016) e uma dissertação de mestrado (Barros-Delben, 2018).

Fatores cognitivos, emocionais e comportamentais, indissociáveis dos estressores comuns presentes em ambientes ICE, são refletidos no desempenho laboral e na saúde dos expedicionários expostos (Andrade \& Souza, 2010; Bannova, 2014; Freitas, 2012; Vanhove, Herian, Harms, Luthans, \& DeSimone, 2014). O estresse é, segundo a Organização Mundial da Saúde (OMS), uma das doenças que mais afeta a saúde do trabalhador (Faro \& Pereira, 2013). Adaptar-se adequadamente a situações de estresse requer estratégias de enfrentamento (coping), construto definido pelo investimento pessoal que recruta meios de minimizar, eliminar ou tolerar fontes de estresse (Lazarus \& Folkman, 1984; Scheier \& Carver, 1992). O estresse é definido por Selye (1975, citado por Ramirez, 2002) como a reação de luta ou fuga do indivíduo decorrente de um estímulo de ameaça ou perigo. Diversas áreas de conhecimento consideram o estresse como uma relação indivíduo-ambiente, integrando aportes de conhecimentos oriundos de pesquisas no âmbito da saúde, da segurança e do trabalho (Faro \& Pereira, 2013; Reis, Fernandes, \& Gomes, 2010). No mundo do trabalho, algumas profissões se destacam como mais suscetíveis ao estresse, como a classe de professores e militares (Maurício, 2016).

Indicadores psicossociais, ocupacionais e físicos permitem a compreensão de possíveis influenciadores para o agravamento ou surgimento de sintomas patológicos (Suedfeld, 2012). Em ambientes ICE, em que há assistência e socorro reduzidos, redes sociais limitadas, sem opções de variações dos estilos de envolvimento pessoal, o estresse assume papel de destaque e tende a gerar prejuízos para os indivíduos envolvidos (Bishop et al., 2010; Paul et al., 2015; Weiss, Feliot, \& Richard, 2011).

A exposição crônica a muitos estressores é comumente observada em missões de longa duração na Antártica, com potenciais impactos negativos na saúde, no desempenho e também em aspectos de segurança do indivíduo e do grupo (Bishop et al., 2010; Hack-Polay, 2012; Kjaergaard, Leon, \& Fink, 2013; Simpson, 2010; Zimmer et al., 2013). Entretanto, durante as operações de verão e temporadas de pesquisa, ocorrem eventos estressores reconhecíveis e padrões, conforme estudos observacionais e coleta de relatos de expedicionários (GongGu, Qian, \& Che, 2011). Logo, o objetivo desse estudo é indicar os principais estressores ambientais, ocupacionais e de relações interpessoais relatados por membros do PROANTAR em missão à Antártica e a variação da percepção destes ao longo da exposição ao ambiente ICE. 


\section{MÉTODO}

\section{Participantes}

Participaram 38 indivíduos, voluntários, selecionados por conveniência e separados em dois grupos, sem repetição de respondentes. O grupo 1 era composto de 15 indivíduos do sexo masculino, com idades entre 26 e 54 anos, cuja coleta de informações foi realizada no início da expedição, a bordo no navio de apoio à missão na Antártica. Já o grupo 2 era formado por 23 indivíduos (15 do sexo masculino e 8 do sexo feminino), com idades entre 24 e 67 anos, os quais forneceram informações no final da expedição. Os pesquisadores que coletaram as informações, embora denominados expedicionários, não fizeram parte dos grupos de respondentes. A função dos militares era oferecer suporte às missões e a dos pesquisadores era realizar pesquisas de cunho diversos, como biológicos, físicos, entre outros. Avaliações físicas e psicológicas, realizadas pelo PROANTAR, qualificaram os participantes como aptos para expedições.

\section{Instrumentos}

Um questionário sociodemográfico foi produzido para caracterizar a amostra. Um roteiro de entrevista estruturado foi construído, com base na literatura internacional (Kjaergaard et al., 2013; Palinkas, Johnson \& Boster, 2004; Paul, 2014) e nas experiências dos pesquisadores em campo, com vistas a abarcar questões relativas à motivação, coping e estresse, emergentes no contexto polar (Zimmer et al., 2013). A decisão por uma entrevista semiestruturada, em detrimento de questionários objetivos sustenta-se pela inexistência de instrumentos validados para o contexto alvo, em língua portuguesa.

Foram selecionados, para as análises neste estudo, 21 itens relacionados ao estresse, de uma entrevista com 38 itens. Inicialmente, foram definidas duas fontes estressoras macro: ambientais e ocupacionais. As fontes de estresse de relações interpessoais foram consideradas, a princípio, como transversais às demais (Nicolas et al., 2013) e, durante a análise de dados, conforme a categorização das respostas ocorria, foi consenso entre os pesquisadores a necessidade de considerá-la como uma a terceira fonte de estresse, buscando verificar variações quanto ao impacto de cada uma delas ao longo da missão.

\section{Procedimento}

A participação foi voluntária e ocorreu a bordo do navio Ary Rongel e Almirante Maximiano em etapas distintas, no início e final de uma missão, após aprovação do projeto pelo Comitê de Ética para Pesquisas com Seres Humanos. Ao considerar a disponibilidade de acesso aos participantes, tendo em vista o espaço de trabalho e residência compartilhados por 24 horas, foi possível realizar as entrevistas nos períodos da manhã, tarde e noite, por vezes, também, durante a madrugada, por não haver espaços privativos, com duração média de $2 \mathrm{~h}$.

\section{Análise de Dados}

Foi realizada uma análise de conteúdo, proposta por Moraes (1999), que segue as seguintes etapas: (a) Preparação das Informações; (b) Unitarização; (c)

Quadro 1

Categorias e subcategorias das fontes de estresse para os expedicionários e suas respectivas definições

\begin{tabular}{|c|c|c|}
\hline Categoria & Subcategoria & Definição \\
\hline \multirow{4}{*}{ Ocupacional } & Privação de sono voluntária. & $\begin{array}{l}\text { Variação do ciclo sono-vigília alterado voluntariamente para concluir suas } \\
\text { atividades, devido a imprevisibilidade quanto a novas oportunidades. }\end{array}$ \\
\hline & Condições precárias de trabalho & Recursos insuficientes para a execução das atividades profissionais. \\
\hline & Turnos de trabalho & $\begin{array}{l}\text { Quantidade de horas de trabalho atribuídas a um profissional em função de } \\
\text { suas responsabilidades. }\end{array}$ \\
\hline & Preocupação com a ocorrência de erros & $\begin{array}{l}\text { Sentimento prévio gerado por possíveis decorrências negativas para si ou para } \\
\text { outros em função de ações inadequadas. }\end{array}$ \\
\hline \multirow{3}{*}{ Ambiental } & Fotoperíodo & Variação da luminosidade natural decorrente das características polares. \\
\hline & Aspectos estruturais & $\begin{array}{l}\text { Aspectos gerais relacionados à estrutura física do navio, insuficiência de } \\
\text { tecnologia e de equipamentos de comunicação. }\end{array}$ \\
\hline & Condições climáticas & $\begin{array}{l}\text { Intempéries naturais ocasionadas pela variação atmosférica e marítima na } \\
\text { região polar. }\end{array}$ \\
\hline \multirow{3}{*}{ Interpessoal } & Sentimento de privação & Sentimento relacionado à falta de pessoa(s), objeto(s), local(is) ou rotina(s). \\
\hline & Interação social & Qualidade da relação estabelecida entre as pessoas pertencentes ao(s) navio(s). \\
\hline & Cultura organizacional & $\begin{array}{l}\text { Conjunto de regras explícitas ou implícitas que orientam as pessoas } \\
\text { pertencentes ao navio. }\end{array}$ \\
\hline
\end{tabular}


Categorização; (d) Descrição e (e) Interpretação. O material coletado foi reunido, incluindo dados do questionário sociodemográfico e da entrevista semiestruturada. A escolha dos dados para a análise priorizou informações associadas ao objetivo da presente pesquisa. As frases registradas nas entrevistas foram adotadas como critério de unidade de análise e tabuladas separadamente para iniciar o processo de classificação. As unidades de análise foram categorizadas como as descritas no Quadro 1. A elaboração das categorias e subcategorias foi realizada previamente à etapa de classificação, com base em estudos internacionais (GongGu et al., 2011; Love \& Bleacher, 2013; Love \& Harvey, 2014; Tafforin, 2009). A contabilização da frequência de frases de cada categoria foi a unidade de quantificação da resposta. A inclusão de conteúdos em categorias foi realizada pelo consenso de dois pesquisadores e de uma avaliação por um revisor neutro, colaborador desta pesquisa. Por fim, descrita frequência percentual de respostas relacionadas a cada categoria e subcategoria, foi verificada a diferença de fontes estressoras na comparação dos dados nas etapas inicial e final da missão.

\section{RESULTADOS E DISCUSSÃO}

As demandas percebidas pelos primeiros desbravadores do continente gelado, com relação direta aos impactos psicofisiológicos, foram melancolia e abuso de álcool (Guly, 2012; Paul et al., 2015; Zimmer et al., 2013). Atualmente, os estudos internacionais focam a definição dos tipos de estressores predominantes no contexto ICE (Bishop et al., 2010; Hack-Polay, 2012; Kjaergaard et al., 2013; Palinkas, Keeton, Shea, \& Leveton, 2011; Paul et al., 2015; Simpson, 2010; Weiss et al., 2011; Zimmer et al., 2013), ratificados nesta pesquisa.

A Tabela 1 apresenta a porcentagem de respostas para cada uma das fontes estressoras no início e final da missão. No início da missão, $23,80 \%$ dos expedicionários indicaram como fonte de estresse os fatores ocupacionais, enquanto $60,71 \%$ destacaram fontes ambientais e $15,47 \%$, fatores interpessoais. Já no fim da missão, $11,94 \%$ dos expedicionários apontaram os fatores ocupacionais como fonte de estresse, $32,08 \%$, os fatores ambientais, e 55,97\%, os fatores interpessoais. Foi observado que a fonte estressora mais citada no início da missão relacionava-se a fatores ambientais e, no final da missão, a fatores interpessoais o que indica que a convivência contínua entre os expedicionários e o ambiente limitado tendem a se transformar em fonte de estresse. É possível que a baixa previsibilidade das condições climáticas e da execução das atividades da missão tenham contribuído pra tornar as relações interpessoais uma fonte de estresse.

As categorias de estressores definidas, juntamente com suas respectivas subcategorias, são discutidas a seguir.

\section{Estressores Ambientais}

Essa categoria é composta por geradores de estresse relacionados à variação de luminosidade natural, aspectos gerais - a exemplo da estrutura física do navio - e intempéries climáticas. Esses estressores, em sua maioria, não apresentam possibilidade de alteração ou controle.

Fotoperíodos. A literatura indica que o excesso de luminosidade no verão e a escassez de luminosidade no inverno afetam os ritmos biológicos (e.g., Claustrat \&
Leston, 2013) e o sono (e.g., Weymouth \& Steel, 2013). Adicionalmente, alguns estudos sugerem que a latitude e altitude afetam o comportamento humano, gerando variações sazonais ou cíclicas em função da luminosidade solar recebida na região, bem como alterações hormonais associadas ao aumento da sintomatologia depressiva e ansiogênica (Cobra, 2009; Flynn-Evans et al., 2016; Zimmer et al., 2013). Testes cognitivos de desempenho podem indicar o nível de impacto do elemento "luz solar" em seres humanos e intervenções com exposição à luz artificial têm se mostrado eficazes no combate a esses sintomas (Cobra, 2008; Weymouth \& Steel, 2013).

Embora o fotoperíodo seja comumente considerado um fator estressor na literatura (e.g., Caballero-Arce, Vigil, \& Benlloch-Marco, 2012; Claustrat \& Leston, 2013; Paul et al., 2015; Eisenstein, 2013), resultado similar não foi encontrado neste estudo (ver Tabela 1). Recomenda-se, no entanto, que projetos futuros realizem a coleta de amostras hormonais, cortisol, melatonina e vitamina $\mathrm{D}$, e aprofundem a avaliação da percepção da sazonalidade.

Aspectos estruturais. A estrutura da embarcação Ary Rongel, diferentemente do Almirante Maximiano, não está preparada para enfrentar condições meteorológicas extremas. Sua estrutura menor gera desconforto e faz com que as pessoas se reúnam nos ambientes de acesso comum, promovendo maior integração, em conformidade com o que prediz o estudo de Caballero-Arce et al. (2012). A viagem de navio é uma das dificuldades para quem vai ao continente gelado por esta via, mas também há inconvenientes para os que optam ou são alocados nos voos de apoio, como falta de horários precisos devido ao clima, bem como gastos elevados com estadias e alimentação. $\mathrm{O}$ aspecto da habitabilidade, termo que concentra as informações das estruturas físicas disponíveis, é importante e pode ser salutar, quando feitos investimentos adequados (Bannova, 2014). Dessa forma, as instalações não devem prover somente o conforto físico, mas também o psicológico (Broughton, 2016).

Aspectos estruturais foram os mais citados entre os estressores ambientais, pelos dois grupos, tanto no início $(90,19 \%)$ quanto no final da missão $(55,31 \%)$, como 
Tabela 1

Fontes de estresse do início e final da missão

\begin{tabular}{|c|c|c|}
\hline \multirow{2}{*}{ Fontes de Estressoras } & \multicolumn{2}{|c|}{ Respostas $(\%$ e $N)$} \\
\hline & Início da Missão & Fim da Missão \\
\hline Ocupacional & $23,80 \%(20)$ & $11,94 \%(35)$ \\
\hline a) Privação de sono voluntária & s.n & $17,14 \%$ \\
\hline b) Condições precárias de trabalho & $55 \%$ & $34,28 \%$ \\
\hline c) Turnos de trabalho & $30 \%$ & $48,57 \%$ \\
\hline d) Preocupação com erros & $15 \%$ & s.n \\
\hline Ambiental & $60,71 \%(51)$ & $32,08 \%(94)$ \\
\hline a) Fotoperíodo & s.n & s.n \\
\hline b) Aspectos estruturais & $90,19 \%$ & $55,31 \%$ \\
\hline c) Condições climáticas & $9,80 \%$ & $44,68 \%$ \\
\hline Interpessoal & $15,47 \%(13)$ & $55,97 \%(164)$ \\
\hline a) Sentimento de privação & $15,38 \%$ & $10,97 \%$ \\
\hline b) Interação social & $69,23 \%$ & $66,46 \%$ \\
\hline c) Cultura organizacional & $15,38 \%$ & $22,55 \%$ \\
\hline Total & $100 \%(84)$ & $100 \%$ \\
\hline
\end{tabular}

Nota: s.n.= sem número (não houve respostas para a fonte estressora em questão)

mostra a Tabela 1. Características da estrutura do navio, da comunicação e de equipamentos de trabalho não variaram no decorrer da missão, o que explica terem sido citadas mais frequentemente nos dois momentos da missão e por grupos distintos. A diminuição na frequência de respostas nessa categoria pode ter decorrido de outras fontes estressoras (e.g., condições climáticas) que assumiram destaque ou se tornaram mais discriminativas.

$\mathrm{O}$ isolamento característico de ambientes ICE (Leveton, 2014) foi transformado com o advento da internet e seu acesso nas estações antárticas ou nos navios. Além disso, foram desenvolvidas linhas de telefone que permitem a comunicação ampliada, ainda que virtual, e promovem a aproximação entre os expedicionários e o mundo exterior. A internet $\mathrm{e}$ o telefone constituem um tipo de suporte estrutural, embora sejam frequentes os atrasos ou interrupções dos sinais, implicando prejuízos ocupacionais que se sobressaem em relação aos prejuízos de contato com a família, como nos envios de relatórios técnicos (Bishop et al., 2010). Muitas vezes visto como um fator salutar, a comunicação com a família e amigos a partir da Antártica pode resultar em uma influência negativa quando eventos problemáticos são transmitidos, como em casos de mortes, acidentes ou doenças graves, comprometendo o desempenho dos membros da equipe e gerando sentimento de impotência àqueles que não podem se deslocar para prestar o apoio que julgam necessário (GongGu et al., 2011; Kjaergaard et al., 2013).

Outro aspecto estrutural mencionado pelos entrevistados foi a privacidade durante a estadia na Antártica. A falta de privacidade e o confinamento, típicos de ambientes ICE (Donoghue, 2016; Schmidt et al., 2015) obrigam as pessoas a interagir, ao menos no início. Entretanto, impede que as pessoas possam recorrer a um local isolado quando assim o desejam (Weiss et al., 2011). Um dos recursos para minimizar o prejuízo da falta de privacidade é, conforme reportado pelos entrevistados, o tratamento acústico adequado, tal como faz a estação franco-italiana (Tafforin, 2009), que conta com uma parte construída para ser silenciosa, onde há quartos, laboratórios e hospital, assim como uma parte em que há ruídos consideráveis, abrigando a casa de máquinas, cozinha e refeitório (Zaganelli \& Alvarez, 2011). Nas instalações brasileiras, contudo, esses recursos estruturais são discutidos e planejados para serem incorporados na nova EACF - com previsão de inauguração em 2020, e nas embarcações recém adquiridas.

Condições climáticas. Diferentemente do esperado no início de uma missão, apenas $9,8 \%$ dos entrevistados apontaram o clima como uma fonte de estresse. No final da missão, entretanto, o clima foi indicado como um fenômeno estressor por quase metade dos participantes (Tabela 1). Alguns transtornos psicofisiológicos são provocados pelo clima, como o Transtorno Afetivo Sazonal, associado ao frio, um estressor natural inerente à missão na Antártica (Færevik, Sandsund, Wiggen, \& Renberg, 2013; Gailliot, 2014; Steinach et al., 2015). Mesmo as roupas fornecidas pelo PROANTAR sendo suficientes e adequadas para o enfrentamento dessa situação, na segunda etapa o uso de uniforme fez emergir o descontentamento de militares quanto à obrigatoriedade de seu uso mesmo nos momentos de folga, com a justificativa de estarem de prontidão constantemente.

A maior parte da amostra reportou eventos negativos da missão, sendo o pior deles a entrada no Drake, região 
do encontro dos oceanos Atlântico e Pacífico, entre o extremo sul do continente americano e Antártica (Resende de Assis, 2015), pois as pessoas passam mal, "mareiam". $\mathrm{O}$ desconforto exige uso de medicamentos para enjoo ou a permanência na cama em posição horizontal, o que alivia os sintomas. A espera em Porto Williams, território chileno, também foi apontada como evento estressor por ser um momento improdutivo, o que vem de encontro com a literatura que trata a subcarga ou a monotonia como aspectos negativos da experiência dos verões antárticos. (Bishop et al., 2010; Landon et al., 2015; Love \& Bleacher, 2013).

O profissional da psicologia no local pode estimular experiências que propiciam saúde, como apontado no trabalho de Suedfeld (2012), exaltando a beleza natural, o mistério, o conforto, a novidade e metas bem estabelecidas, desviando o foco dos estressores patogênicos. Por exemplo, entre os aspectos positivos de estar no ambiente antártico, mencionados pelos entrevistados, estavam as paisagens e o dia em que o mar surgiu congelado, indicando, finalmente, a chegada na Antártica. Todavia, não são todos que despertam para esses aspectos sem estímulos externos, seja um colega insistindo para ver a paisagem, um profissional ou um protocolo estabelecido que motive tal comportamento. A tendência atual é de estudos com observação dos aspectos salutogênicos, melhorando consideravelmente o humor e o clima entre as pessoas (Zimmer et al., 2013).

\section{Estressores Ocupacionais}

A fonte estressora ocupacional mais frequente no início da missão relacionava-se às condições precárias de trabalho (55\%), enquanto no final da missão, aos turnos de trabalho $(48,57 \%)$, conforme indicado na Tabela 1 . As exigências do trabalho, aliadas ao cansaço e sobrecarga, podem justificar a variação na percepção dos expedicionários nos dois momentos da missão. Adicionalmente, na perspectiva dos entrevistados, o estresse ocupacional é o fator de menor impacto ao final da missão (Tabela 1), momento em que se torna menos relevante do que a fonte menos expressiva de estresse no início da expedição (i.e., fatores interpessoais). Considerando o crescimento de questões de ordem pessoal com o decorrer do tempo, é possível que os expedicionários enfrentem os problemas ocupacionais como inevitáveis, assim como os ambientais, acostumando-se a eles, o que propiciaria a adoção de estratégias de coping focadas na emoção (Lazarus \& Folkman, 1984).

Com base na evidência de que pessoas mais otimistas tendem a apresentar um coping ativo, o qual focaliza a ação, pode-se dizer que os pessimistas são caracterizados por evitar as fontes estressoras quando julgam não haver recursos para resolver as situações dispostas, logo, se caracterizam pelo coping que foca a emoção. Esse tipo de coping pode ser funcional ou disfuncional (Lazarus \& Folkman, 1986), tal qual a conclusão de um dos estudos empíricos recentes realizados no Brasil com esse público (Pereira et al., 2016).
Um exemplo de coping focalizado na emoção disfuncional é o abuso de álcool, um problema recorrente em regiões polares e reportado pelos entrevistados como uma questão urgente.

Privação de sono voluntária. A privação de sono, especialmente quando voluntária, surgiu como informação espontânea nas entrevistas do início da missão, embora não constasse nas questões do roteiro elaborado, sendo, portanto, introduzida no instrumento utilizado no final. Nesta ocasião, a privação de sono voluntária foi apontada como uma fonte de estresse por 17,14\% dos entrevistados (Tabela 1).

Pesquisadores e militares na Antártica ficam expostos ao ambiente externo por muitas horas seguidas (Corbett et al., 2012; Kjaergaard et al., 2013; Paul, 2014; Vanhove et al., 2014), pois algumas tarefas exigem a permanência fora das instalações (navios, estação, refúgios) para compensar atrasos no planejamento provocados por condições climáticas. O tempo disponível para a realização das atividades militares ou de pesquisa depende da meteorologia e das ordens dos voos e navios de apoio. Há pessoas que ficam mais de $18 \mathrm{~h}$ trabalhando ininterruptamente para aproveitar o "tempo bom" e, voluntariamente, alteram seu ciclo sonovigília, pois não sabem quando terão novas oportunidades. Entretanto, o principal responsável pelas poucas horas de sono na Antártica, pela amostra do PROANTAR, não é o fator ambiental, e sim, o fator ocupacional aliado a fatores interpessoais (Mallis e DeRoshia,2005).

Condições precárias de trabalho. Esse fator foi apontado como uma fonte de estresse por $55 \%$ dos respondentes no início da missão e por $34,28 \%$ dos respondentes no final. Considerações acerca das condições de trabalho e os desafios enfrentados para exercer as atividades transformam diversos aspectos ambientais em estressores ocupacionais (Bishop et al., 2010). Há prazos para o cumprimento das tarefas, mas condições climáticas dificultam a execução dessas tarefas, ocasionando atrasos e requerendo ajustes no cronograma. Viagens são adiadas ou canceladas quando o tempo não permite pousos em pistas com neve ou navegação em meio a blocos de gelo no mar. O mal tempo também prejudica a qualidade do sinal de internet, sendo esse o aspecto negativo mais citado pelos respondentes, um problema também reconhecido em outros estudos (Love \& Bleacher, 2013; Tothill \& Martin, 2012). Essa baixa qualidade da internet prejudica trabalhos, como o envio de relatórios ou solicitações. A utilização desse serviço durante a madrugada foi uma alternativa apontada pelos entrevistados, algo que prolonga o expediente e eleva os níveis de fadiga relatados.

Há decaimento do desempenho laboral em caso de funcionamento ruim dos equipamentos e perigos de lesão em função de mudanças de clima durante as atividades (GongGu et al., 2011). Alguns trabalhos na Antártica requerem precisão motora fina e, às vezes, é preciso tirar as luvas, o que pode provocar queimaduras pelo frio. Um estudo no Ártico (Norlander, Miller, \& Gard, 2015) indicou 
a urgência de investimentos em equipamentos de proteção individual, o que tende a evitar acidentes, como escorregões, ou congelamentos. Destaca-se que as vestimentas nem sempre são apropriadas para a locomoção ou manuseamento de instrumentos e exigem sincronismo entre ações em nível logístico e operacional.

Especificamente em expedições embarcadas (i.e., as pessoas permanecem nos navios), durante as manobras do meio de transporte e residência compartilhada, a atenção é voltada para os acidentes em potencial. A adaptação à rotina de embarcados foi mencionada pelos participantes pesquisadores do presente estudo como dificultosa, e entre os militares, o problema residiu na falta de um manual ou histórico de todas as funções.

Turnos de trabalho. Noções de hierarquia, funções e especialidades, bem como as distribuições de responsabilidades de tarefas e atividades em uma instituição sem divisões hierárquicas geram mais colaboração nos trabalhos (Bishop et al., 2010; Weiss et al., 2011). É fundamental entender a rotina, os meios de comunicação externos para apoio, comportamentos em situações de crise, como incêndios, tempestades, desabamentos, entre outros (Bishop et al., 2010; Burns \& Sullivan, 2000).

Os militares devem estar à disposição em tempo integral (Umann \& Lautert, 2016), delegar tarefas e cumprir turnos, com horários estabelecidos, inclusive na madrugada. Há, também, "encargos colaterais", ou seja, os mais antigos sempre assumem os cargos daqueles que por alguma razão se ausentam. Entre os pesquisadores há uma tradição de se capacitar para exercer todas as tarefas necessárias para a coleta de dados, de modo a aumentar as chances de que a coleta seja realizada no tempo disponível nas missões (Tothill \& Martin, 2012),

Esses aspectos, além de gerar uma sobrecarga de trabalho, prolongam o estado de alerta. Daí não ser surpreendente que $48,57 \%$ dos respondentes tenham apontado, no final da missão, os turnos de trabalho como uma fonte de estresse, em contraposição ao início da missão, quando não mais do que $30 \%$ dos respondentes fizeram o mesmo.

Dentre os aspectos laborais positivos em contextos antárticos, destaca-se a formação de equipes heterogênicas (Landon et al., 2015), tanto em termos culturais quanto de gênero. Essa formação facilita ajustes psicológicos e aumenta a motivação pelo convívio interpessoal saudável, assim como a colaboração nas atividades requeridas, visto que funções são compartilhadas e todos devem estar aptos a suprir as demandas (Zimmer et al., 2013).

Preocupação sobre a possibilidade de ocorrência de erros nas atividades. Um sentimento antecipatório de consequências negativas para si ou para outros, em função de ações inadequadas, foi identificado como uma fonte estressora, por uma pequena parcela dos respondentes $(15 \%)$, mas apenas no início da missão. Pode-se inferir que esse estresse é ocasionado pela ansiedade e expectativa frente ao desconhecido e pelo empenho em realizar uma expedição sem maiores problemas. Além disso, há o fato de que no início da missão, outros aspectos do contexto ainda não se revelaram como fontes de estresse, o que permite que as preocupações com o sucesso da expedição prevaleçam, deixando o indivíduo em estado de alerta permanente (Ramírez, 2002; Umann \& Lautert, 2016) devido aos riscos.

Todas as fontes de estresse da categoria Estressores Ocupacionais combinadas tendem a culminar na maior frequência de erros na execução de tarefas, conforme os paralelos traçados com profissionais que trabalham em turnos, (Halsey \& Stroud, 2012; Paul et al., 2015; Premkumar et al., 2013; Rui et al., 2015).

\section{Estressores Interpessoais}

Enquanto os estressores ambientais e ocupacionais diminuíram ao longo da missão, os interpessoais apresentaram uma dinâmica crescente (Tabela 1). As tensões nas expedições antárticas surgem, segundo os entrevistados e com base na literatura (Donoghue, 2016; Færevik et al., 2013; Nicolas et al., 2013), em decorrência de confinamento, isolamento, falta de comunicação, convivência forçada com grupos reduzidos e relações de poder. Foi afirmado também medo ou receio da experiência em si, por ser uma área inóspita e com muitos perigos.

Sentimento de privação. Essa subcategoria foi apontada como uma fonte de estresse por $15,38 \%$ e $10,97 \%$ dos entrevistados, no início e no final da missão, respectivamente. As principais dificuldades apontadas pelos expedicionários, principalmente pelos militares, que ficam seis meses embarcados ou um ano na Estação, foi o afastamento da família por longos períodos. Os pesquisadores, por sua vez, ficam de um a dois meses nas missões. Devido à frequência do uso da palavra "saudade", considera-se este um item específico da cultura brasileira, já que o termo se refere ao sentimento de falta da casa, dos amigos e da família, por exemplo. Nota-se que há poucas referências sobre saudades na literatura internacional no contexto antártico (GongGu et al., 2011; Smith, Kinnafick \& Saunders, 2017) e que o termo mais aproximado utilizado é homesickness, embora não tão amplo como a definição da palavra utilizada pelos brasileiros (Gonçalves, 2010).

O sentimento denominado "saudade" pode ser entendido como um estado cognitivo-emocional-motivacional (Ferraz \& Pereira, 2002; Fisher \& Hood, 1987) em que há sofrimento psíquico em decorrência da relocação de casa para viver em novos ambientes, havendo frequentemente sentimentos de angústia e de preocupação relacionados ao lar (Fisher \& Hood, 1987; Van Tilburg, Vingerhoets, \& Van Heck, 1996). Quanto ao sentimento homesickness, profissionais podem 
apresentar desinteresse no trabalho e falta de concentração (Hack-Polay, 2012), acompanhados por uma redução no sentimento de pertença ou mesmo de identificação com a missão, situação verificada entre os participantes da presente pesquisa. Alguns, entretanto, reportaram que embora sentissem saudades e estivessem sofrendo por isso, consideravam-se também culpados pelo fato de estarem desfrutando a experiência e sentindo-se privilegiados.

A distância e o isolamento de outras comunidades humanas são desafios a mais para os expedicionários, principalmente no inverno, com noites que duram $24 \mathrm{~h}$ por dia durante meses, restringindo o espaço das pessoas aos conteiners e estações fixadas ou interior de embarcações e acampamentos no verão (Cobra, 2009; Corbett et al., 2012; Kjaergaard et al., 2013; Paul, 2014). Porém, o isolamento absoluto na Antártica é relativo uma vez que, apesar de geograficamente isolados, em algumas estações circulam mais de 1000 pessoas. Não obstante, nas áreas de atuação do PROANTAR há fluxos distintos, assim considerado nas respostas às entrevistas.

O isolamento autônomo, ou seja, que se estabelece por iniciativa do sujeito como resposta adaptativa disfuncional ao isolamento e que se caracteriza por separação geográfica e social inicial, transforma-se em reclusão voluntária, afetando os expedicionários em uma produção crônica de solidão (GongGu et al., 2011; Simpson, 2010). Mesmo que o suporte social esteja disponível no local, a falta de conhecimentos que instrumentalizem o expedicionário a lidar com os problemas que surgem ou incentivando-o a compartilhar suas angústias influencia seu comportamento até mais que os estressores ambientais. Não raro foram as menções de que algumas pessoas preferiam se isolar e que isso preocupava os demais.

Cultura organizacional. Entendida como um padrão de pressupostos e de formas de perceber, pensar e sentir desenvolvido por um grupo de pessoas com o objetivo de funcionarem suficientemente bem e de aprenderem a lidar com situações externas e interações internas do grupo, podendo ser ensinado a novos membros (Schein, 1992), a cultura organizacional foi apontada como uma fonte estressora por $15,38 \%$ dos respondentes no início da missão e por $22,55 \%$ ao final. Os aspectos culturais militares, de pesquisadores e de regiões polares se atravessam nessa compreensão.

As organizações com características militares, como é o caso das missões antárticas, são pautadas em dois preceitos básicos: hierarquia e disciplina. O modelo de organização militar, que tende a assumir uma estrutura burocrática, com ênfase em aspectos formais da organização e pouca consideração pelos trabalhadores em nível operacional, acabou gerando uma visão parcial do trabalho da Marinha do Brasil pelos pesquisadores selecionados, um resultado consistente com aquele relatado por Andrade e Souza (2010). Os membros da organização geralmente compreendem como mais "justo" o voto da maioria e, assim, singularidades não são contempladas (GongGu et al., 2011). A ocorrência dessa visão não faz parte do escopo desse trabalho, mas é possível que o padrão de conduta militar, regido por regras explícitas ou não, seja considerado como estressor para os expedicionários, em especial os civis, devido à não familiaridade.

A cultura estabelecida dentro do navio, por se tratar de um ambiente restrito e composto por pessoas de diferentes locais, também pode influenciar no desenvolvimento de estresse (Faro \& Pereira, 2013; Jitwasinkul et al., 2016; Suedfeld, 2012). A fim de minimizar esse estímulo estressor entre os expedicionários, sínteses sobre as áreas de formação ou atuação profissional de militares e pesquisadores permitiriam um entendimento ampliado das missões na Antártica e das funções dos prestadores de apoio logístico, visando a redução dos impactos negativos relacionados à interação social.

Interação social. Aspectos da interação social foram considerados como as maiores fontes de estresse da categoria interpessoal, no início $(69,23 \%)$ e no final $(66,46 \%)$ da missão, o que pode indicar um elemento que requer maior atenção nesse contexto.

O caráter de "internato", compartilhamento de trabalho e residência $24 \mathrm{~h}$ por dia, inclusive nos momentos de "folga", contribui para o desgaste contínuo nas relações, intensificado pelas exigências do pouco espaço e falta de privacidade, e até mesmo para a euforia observada nos expedicionários. Além disso, nos navios, muitas vezes é necessário que os expedicionários realizem atividades que não estão diretamente relacionadas com suas especialidades (Hetherington \& Mearns, 2006), o que pode gerar insegurança e sensibilidade à crítica dos colegas. Outras variáveis que interferem na dinâmica interpessoal são a falta de confiança no colega, diferenças de valores e depreciação da conduta do outro (Baasch, Amorim \& Cruz, 2015; Santos \& Cardoso, 2010). Esses são indicadores da dificuldade do trabalho em equipe nas atividades que exigem a divisão de tarefas. Como consequência, aumentam as dificuldades de funcionamento grupal e há diminuição da função do grupo como potencializador de suporte social (Landon et al., 2015).

Foi mencionado pelos entrevistados como um aspecto positivo da interação social, o acolhimento do grupo-base na Estação, quando receberam os primeiros pesquisadores após a temporada de inverno, período de maior isolamento durante toda a missão. Nessa ocasião, os expedicionários tiveram oportunidade de expor suas emoções, sua de satisfação em encontrar visitantes sem filtros. O compartilhamento de emoções costuma ser feito em situações específicas do trabalho (D'Incao \& Gastaud, 2015; Love \& Bleacher, 2013), porém o suporte social não alcança níveis pessoais que garantam um espaço confortável ou seguro para expor aspectos psicológicos. Com os meios de comunicação disponíveis, há a preferência para compartilhamento com familiares e amigos, via online. Em atividades de lazer, normalmente com consumo de bebidas alcoólicas - que são um costume em regiões polares, mas que podem ocasionar problemas (Nicolas et al., 2013) - há maior propensão à 
comunicação entre o grupo antártico, promovida com o intuito de revelar emoções (Palinkas et al., 2004).

Com a exposição ao ambiente ICE, são observadas evidências de diminuição do desejo de procurar pelo suporte social, em diversos níveis, em uma espécie de conformidade adaptada ao contexto. As relações dos grupos formados tendem a se deteriorar com o acúmulo de tolerância, emergindo mudanças extremas de humor, conforme relatado por GongGu et al. (2011), Kjaergaard et al. (2013), Paul (2014) e Weiss et al. (2011). Similarmente, Rodrigues e Ferreira (2011) encontraram uma correlação negativa e estatisticamente significativa entre relação interpessoal e nível de estresse de enfermeiros de UTI. Em um estudo semelhante sobre fatores estressores na atividade de médicos, destaca-se o relacionamento com a equipe, com os colegas e com a chefia como variáveis significativas para o estabelecimento de estressores, o que pode ocorrer devido à alta divisão no processo de trabalho (Santos et al., 2011).

\section{CONSIDERAÇÕES FINAIS}

Este mapeamento na missão de verão do PROANTAR identificou estressores que contribuem para a redução da qualidade de vida e do desempenho das atividades laborais, em etapas distintas da expedição, ressaltando, porém, a presença de fatores peculiares à cultura brasileira tais como a expressão de saudade e a ausência da percepção de privação de sono. Recomenda-se mais investimentos nesse campo, principalmente no cenário nacional, considerando os incentivos de órgãos fiscalizadores da pesquisa científica na Antártica, as evidências encontradas sobre fontes de estresse e seu impacto no ser humano e as abordagens multidisciplinares para a captação dos fenômenos no ambiente ICE.

Contribuições de estudos psicológicos na Antártica se dirigem à seleção e preparação de pessoal, treinamento e desenvolvimento de pessoas, intervenções in loco e ações de prevenção ao surgimento de demandas. A participação da Psicologia no PROANTAR tem importância reconhecida, principalmente devido aos trabalhos do Serviço de Seleção do Pessoal da Marinha (SSPM), que realiza investigações quanto à atenção dos aspectos de militares que formam o grupo-base de guarnição da Estação. Entretanto, houve limitações para o presente estudo, especialmente por ser uma fase de exploração e reconhecimento do campo, exigindo ajustes na pesquisa diante das condições dispostas, bem como frequentes alterações de cronograma.

Para manter a saúde dos expedicionários frente aos estímulos estressores das interações sociais, é pertinente que a organização planeje ações que antecipem o surgimento desse tipo de estímulo estressor. Como exemplos disso, há o desenvolvimento de grupos terapêuticos com temas considerados estressores e aversivos levantados em missões anteriores; a criação, na etapa de preparação para a missão, da receptividade dos seus envolvidos, especialmente entre os oficiais, que são os líderes dos grupos; proposição de atividades em conjunto em espaços laborais com o intuito de aumentar a confiança e o vínculo entre todos os profissionais do navio.

É preciso entender a estrutura organizacional do PROANTAR, o perfil das pessoas nas missões e os recursos disponíveis, com resumos das atividades dos profissionais. Sugere-se a facilitação da proximidade entre os grupos de expedicionários, como a familiarização das rotinas em situação embarcada por civis. Um panorama das formações e das pesquisas realizadas pelos cientistas pode propiciar melhor convívio e maior colaboração na execução das atividades e enfrentamento das condições adversas do local.

Diante dos resultados encontrados nesta pesquisa, é importante criar estratégias que diminuam o sentimento de privação de fatores gratificantes, como família, local onde vive ou qualquer estímulo que desperte o sentimento de saudade. Para tal, investimentos em meios de comunicação e ampliação de espaços nos quais os sujeitos possam expor suas angústias, por exemplo, se apresentam como ações minimizadoras das consequências do estresse para esses profissionais.

Compreender fenômenos psicológicos envolvidos em ambientes ICE é importante para: (a) contribuir com o preenchimento da lacuna na literatura sobre a Psicologia em ambientes ICE, especialmente a nacional; (b) capacitar profissionais que atuam nesse tipo de ambiente em termos de prevenção e enfrentamento de fontes estressoras existentes em ambientes ICE; (c) auxiliar gestores, seja de organizações envolvidas em projetos na Antártica, seja do próprio navio, pois o conhecimento produzido acerca dos fenômenos psicológicos ajudam na tomada de decisão, promovendo manejos de planejamento, preparação e ações de prevenção menos custosas que ações de "posvenção". Finalmente, a sociedade como um todo é beneficiada já que o conhecimento produzido em pesquisas na Antártica é aplicado em populações que enfrentam situações extremas de temperatura, confinamento e risco similares aos encontrados na Antártica. 


\section{REFERÊNCIAS}

Andrade, E. R. D., \& Souza, E. R. D. (2010). Autoestima como expressão de saúde mental e dispositivo de mudanças na cultura organizacional da polícia. Psicologia Clínica, 22(2), 179-195. Retirado de http://dx.doi.org/10.1590/S010356652010000200012

Baasch, D., Amorim, L., \& Cruz, R. M. (2015). Qualidades psicométricas de instrumentos de resiliência para adultos. Revista Borges, 5(1), 38-53.

Baldrighi, R. D. M. (2016). Antártida: Uma análise históricocomparada das presenças brasileira e argentina no continente gelado. Trabalho apresentado no III Seminário de Relações Internacionais, Centro Universitário Tabosa Almeida, Caruaru, PE.

Bannova, O. (2014). Extreme environments-design and human factors considerations (Dissertação de Mestrado). University of Technology, Gothenburg, Suécia.

Barros-Delben, P. (2018). Comportamento seguro em expedicionários militares do Programa Antártico Brasileiro (PROANTAR) (Dissertação de Mestrado). Universidade Federal de Santa Catarina, Florianópolis, SC.

Barros-Delben, P., Pereira, G. K. \& Cruz, R. M. (2017). The importance to the health and safety of Brazilian Expeditionaries with the Air Cargo Launch Operations to the Brazilian Antarctic Station in the winter. Trabalho apresentado na APECS International Online Conference 2017 "Outside the Box".

Barros-Delben, P., Sombrio, L. S., Melo, H. M., Pereira, G. K., \& Cruz, R. M. (2015). Estresse: Potenciais prejuizos cognitivos em expedicionários antárticos. Trabalho apresentado na VI Reunião do IBNEC e 1st Brazilian Meeting of the Human Behavior and Evolution Society, Gramado, RS.

Bishop, S. L., Kobrick, R., Battler, M., \& Binsted, K. (2010). FMARS 2007: Estresse and coping in an arctic Mars simulation. Acta Astronautica, 66(9),1353-1367. doi:10.1016/j. actaastro.2009.11.008

Broughton, H. (2016). Polar research facilities: Living in isolation. Trabalho apresentado na 46th International Conference on Environmental Systems, Viena, Áustria.

Burns, R., \& Sullivan, P. (2000). Perceptions of danger, risk taking, and outcomes in a remote community. Environment and Behavior, 32(1), 32-71.

Caballero-Arce, C., Insausti, A. V. de, \& Marco, J. B. (2012). Lighting of space habitats: Influence of color temperature on a crew's physical and mental health. In 42nd International Conference on Environmental Systems. doi.org/10.2514/6.2012-3615

Claustrat, B., \& Leston, J. (2013). Melatonin: Physiological effects in humans. Neurochirurgie, 61(2), 77-84.

Cobra, G. D. O. (2008). Psicologia de grupos: Pesquisadores em isolamento e confinamento na Antártica (Tese de Doutorado). Retirado de http://www.arca.fiocruz.br/handle/icict/ 4335.

Cobra, G. O. (2009). Psicologia Polar. Formação Profissional e Compromisso Social da Psicologia, 2(2), 70-80. Retirado de http://www.ufrrj.br/seminariopsi/2009/boletim 2009-2/ cobra.pdf

Corbett, R. W., Middleton, B., \& Arendt, J. (2012). An hour of bright white light in the early morning improves performance and advances sleep and circadian phase during the Antarctic winter. Neuroscience Letters, 525(2), 146-151. doi: 10.1016/j. neulet.2012.06.046

D’Incao, D. B., \& Gastaud, M. B. (2015). Investigando a associação de sintomas e queixas depressivas com as estações do ano. Fractal: Revista de Psicologia, 27(2), 152-159.
Donoghue, M. (2016). Urban design guidelines for human wellbeing in Martian settlements (Tese de Doutorado). University of Washington, Seattle, WA.

Eisenstein, M. (2013). Chronobiology: Stepping out of time. Nature,497(7450), S10-S12.

Færevik, H., Sandsund, M., Wiggen, Ø., \& Renberg, J. (2013). Arctic weather protection, health effects, monitoring systems and risk assessment (Report nr. SINTEF). Retirado de http://www.scielo.mec.pt/scielo.php?script=sci arttext\&pid=S1645-008620130001 00007\&lng=pt\&tlng=pt.

Faro, A. \& Pereira, M. E. (2013). Medidas do estresse: Uma revisão narrativa. Psicologia, Saúde \& Doenças, 14(1), 101-124. Retirado de http://www.scielo. mec.pt/scielo.php?script=scia rttext\&pid=S1645-0862013000100007

Ferraz, M. F., \& Pereira, A. S. (2002). A dinâmica da personalidade e o homesickness (saudades de casa) dos jovens estudantes universitários. Psicologia, Saúde \& Doenças, 3(2), 149-164. Retirado de http://www.scielo.mec.pt/scielo.php?script=sci arttext\&pid=S1645-00862 002000200004\&lng=pt\&tlng=pt.

Feuerecker, M., Crucian, B., Salam, A. P., Rybka, A., Kaufmann, I., Moreels, M., Quintens, R., Schelling, G., Thiel, M., Baatout, S., Sams, C., \& Choukèr, A. (2014). Early adaption to the antarctic environment at dome C: Consequences on stresssensitive innate immune functions. High Altitude Medicine \& Biology, 15(3), 341-348.

Fisher, S., \& Hood, B. (1987). The estresse of the transition to university: A longitudinal study of psychological disturbance, absent-mindedness, and vulnerability to homesickness. British Journal of Psychology, 78, 425-441. doi: 10.1111/j.20448295.1987.tb02260.x

Flynn-Evans, E., Gregory, K., Arsintescu, L., \& Whitmire, A. (2016). Risk of performance decrements and adverse health outcomes resulting from sleep loss, circadian desynchronization, and work overload (Evidence Report). Retirado do sítio da NASA: https://ntrs.nasa.gov/archive/nasa/casi.ntrs.nasa. gov/20160003864.pdf

Freitas, M. E. (2012). Lições organizacionais vindas da Antártica. Revista de Administração Pública,46(4), 915-937. doi. org/10.1590/S0034-76122012000400002

Gailliot, M. T. (2014). An assessment of the relationship between self-control and ambient temperature: A reasonable conclusion is that both heat and cold reduce self-control. International Review of Social Sciences and Humanities, 8, 149-193.

Gandra, R. M. (2013). Geopolítica antártica no limiar do século XXI:A definição de um projeto estratégico-cientifico para o Brasil na Antártica (Tese de Doutorado). Universidade Federal do Rio Grande do Sul, Porto Alegre, RS.

Gonçalves, L. M. C. (2010). Saudades de casa, depressão e personalidade em estudantes universitários (Dissertação de Mestrado). Retirado de http://repositorio.ismt.pt/handle/ $123456789 / 268$.

GongGu, Y. A. N., Qian, Y. E., \& Che, T. A. N. G. (2011). Adaptation and coping strategies in Chinese Antarctic Expeditioners' winter-over life. Advances in Polar Science, 22(2), 111-117. doi: 10.3724/SP.J.1085.2011.00110

Gonçalves, L. M. C. (2010). Saudades de casa, depressão e personalidade em estudantes universitários (Dissertação de Mestrado). Retirado de http://repositorio.ismt.pt/handle/ $123456789 / 268$.

Guly, H. R. (2012). Psychology during the expeditions of the heroic age of Antarctic exploration. History of Psychiatry, 23(2), 194-205.

Hack-Polay, D. (2012). When home isn't home - A study of homesickness and coping strategies among migrant workers 
and expatriates. International Journal of Psychological Studies, 4(3). doi: 10.5539/ijps.v4n3p62

Halsey, L. G. \& Stroud, M. A. (2012). 100 years since Scott reached the Pole: A century of learning about the physiological demands of Antarctica. Physiological Reviews, 92(2). http://dx.doi. org/10.1152/physrev.00031.2011

Hetherington, C., Flin, R., \& Mearns, K. (2006). Safety in shipping: The human element. Journal of Safety Research, 37(4), 401411. https://doi.org/10.1016/j.jsr.2006.04.007

Jesus, D. T., \& Souza, H. T. (2007). As atividades da Marinha do Brasil na Antártica. O Ecologia Brasiliensis, 11(1), 7-13. Retirado de https://dialnet.unirioja.es/descarga/ articulo/ 2685 346. pdf

Jitwasinkul, B., Hadikusumo, B. H., \& Memon, A. Q. (2016). A Bayesian Belief Network model of organizational factors for improving safe work behaviors in Thai construction industry. Safety Science, 82, 264-273.

Kjaergaard, A., Leon, G. R. \& Fink, B. A. (2013). Personal challenges, communication processes, and team effectiveness in military special patrol teams operating in a polar environment. Environment and Behavior, 46(6), 644-666. doi: $10.1177 / 0013916513512834$

Landon, L. B., Vessey, W. B., \& Barrett, J. D. (2015). Risk of performance and behavioral health decrements due to inadequate cooperation, coordination, communication, and psychosocial adaptation within a team (Evidence Report). Retirado do sítio da NASA: https://ntrs.nasa.gov/archive/nasa/ casi.ntrs.nasa.gov/20150016963.pdf

Lazarus, R. S., \& Folkman, S. (1984). Stress, appraisal, and coping. New York: Springer Publishing Company.

Leveton, L. B. (2014). Review of isolated, confined extreme environment studies. Trabalho apresentado no $6^{\circ}$ International Congress on Medicine and Space in Extreme Environments, Berlim, Alemanha.

Love, S. G., \& Bleacher, J. E. (2013). Crew roles and interactions in scientific space exploration. Acta Astronautica, 90(2), 318-331.

Love, S. G., \& Harvey, R. P. (2014). Crew autonomy for deep space exploration: Lessons from the Antarctic search for meteorites. Acta Astronautica, 94(1), 83-92.

Madureira Júnior, J. (2014). Antártica: A importância do apoio logístico das Forças Armadas à pesquisa científica. Coleção Meira Mattos, 8(31), 49-58.

Mallis, M. M., \& de Roshia, C. W. (2005). Circadian rhythms, sleep, and performance in space. Aviation, space, and environmental medicine, 76(6), B94-107. Retirado de http://www.m3alertness. com/wp-content/uploads/2014/01/SleepSpace.pdf

Maurício, P. M. C. (2016). O trabalho por turnos e suas consequências nos trabalhadores: o caso de técnicos de reparação/manutenção num centro de finishing (Tese de Doutorado). Instituto Politécnico de Setúbal, Setúbal, Portugal.

Melo, H. M., Barros-Delben, P., Sombrio, L. S. \& Cruz, R. M. (2015). Alterações cognitivas em decorrência de prejuizos da qualidade de sono: Trabalho em turnos. Trabalho apresentado no III Congresso Sul-Brasileiro de Cognição: Neuropsicologia e Sono e I Simpósio de Neurociência e Sono, Florianópolis, SC.

Moraes, R. (1999). Análise de conteúdo. Revista de Educação, 22(37), 7-32.

Nicolas, M., Sandal, G. M. N., Weiss, K. \& Yusupova, A. (2013). Mars-105 study: Time-courses and relationships between coping, defense mechanisms, emotions and depression. Journal of Environmental Psychology 35, 52-58.

Norlander, A., Miller, M., \& Gard, G. (2015). Perceived risks for slipping and falling at work during wintertime and criteria for a slip-resistant winter shoe among Swedish outdoor workers. Safety Science, 73, 52-61.

Palinkas, L. A., Johnson, J. C., \& Boster, J. S. (2004). Social support and depressed mood in isolated and confined environments.
Acta Astronautica, 54(9), 639-647. Retirado de http://dx.doi. org/ 10.1016/S0094-5765(03)00236-4

Palinkas, L. A., Keeton, K. E., Shea, C., \& Leveton, L. B. (2011). Psychosocial characteristics of optimum performance in isolated and confined environments (Evidence Report). Retirado do sítio da NASA: http://ston.jsc.nasa.gov/collections/ trs/ techrep/TM-2011-216149.pdf

Paul, F. U. J. (2014). Review on Psychological test for personnel selection in long duration mission to extreme environment. The International Journal of Indian Psychology, 2(1), 121-127. Retirado de http://oaji.net/articles/2014/1170-1416042637.pdf

Paul, M. A., Love, R. J., Hawton, A., Brett, K., McCreary, D. R. \& Arendt, J. (2015), Light Treatment Improves Sleep Quality and Negative Affectiveness in High Arctic Residents During Winter. Photochemistry and Photobiology, 91, 567-573. doi: 10.1111/php. 12418

Pereira, G. K., Barros-Delben, P., Melo, H. M., Mendonça, S. A., \& Cruz, R. M. (2016). Estratégia de coping de tripulantes aeronavais no início e ao final de expedição à Antártica. Trabalho apresentado no XXV Encontro Brasileiro de Psicologia e Medicina Comportamental, Foz do Iguaçu, PR.

Premkumar, M., Sable, T., Dhanwal, D. \& Dewan, R. (2013). Vitamin D homeostasis, bone mineral metabolism, and seasonal affective disorder during 1 year of of Antarctic residence. Arch Osteoporos, 8(1-2):129. doi: 10.1007/s11657-013-0129-0

Ramírez, H. D. C. (2002). Estresse no cotidiano de trabalho de bombeiros de Santa Catarina: Entre as atividades profissionais e as exigências da organização (Dissertação de Mestrado). Retirado de http://repositorio.ufsc.br/xmlui/ handle/123456789/82462

Reis, A. L. P. P., Fernandes, S. R. P. \& Gomes, A. F. (2010). Estresse e fatores psicossociais. Psicologia, Ciência e Profissão, 30(4), 712-725. http://dx.doi.org/10.1590/S1414-989320 10000400004

Resende de Assis, L. G. (2015). A colonização científica brasileira da Antártida: Notas para pensar antropologicamente. Cuadernos de Antropología, 13, 65-80.

Rodrigues, V. M. C. P., \& Ferreira, A. D. S. (2011). Factores generadores de estrés en enfermeros en unidades de terapia intensiva. Revista Latino-Americana de Enfermagem, 19(4), 1025-1032. Retirado de http://www.scielo.br/pdf/rlae/v19n4/ es 23.pdf

Rui, M. de., Middleton, B., Sticca, A., Gatta, A., Amodio, P., Skene, D. J., \& Montagnese, S. (2015). Sleep and circadian rhythms in hospitalized Patients with decompensated cirrhosis: effect of light therapy. Neurochemical Research, 40, 284-292. Retirado de http://dx.doi.org/ 10.1007/ s11064-014-1414-z

Santos, A. F. de O., \& Cardoso, C. L. (2010) Profissionais de saúde mental: Estresse e estressores ocupacionais em saúde mental. Psicologia em Estudo, 15(2), 245-253. Retirado de http:// dx.doi.org/10.1590/S1413-73722010000200003

Santos, C. L. M., Rodrigues, C. L. P., Silva, L. B., Bakke, H. A., Leite, A. S. M., \& Leal, M. M. A. (2011). Fatores de estresse na atividade de médicos em João Pessoa (PB, Brasil). Production, 21(1), 181-189. Retirado de http://www.scielo.br/ pdf/prod/2011nahead/AOP 200811118.pdf

Scheier, M. F., \& Carver, C. S. (1992). Effects of optimism on psychological and physical well-being: Theoretical overview and empirical update. Cognitive Therapy and Research, 16(2), 201-228. doi: 10.1007/BF01173489

Schein, E. H. (1992). Organizational culture and leadership $\left(2^{\text {nd }}\right.$ ed.). San Francisco, CA: Jossey-Bass.

Schmidt, L. L., Landon, B., \& Patterson, H. (2015). A model of psychosocial factors for long-duration spaceflight exploration missions (Technical Report). Retirado do sítio da NASA: https://ston.jsc.nasa.gov/collections/TRS/ techrep/TM-2015218582.pdf 
Silva, F. C. C., Cabral, J. C. C. R., \& Zimmer, M. (2014). Produção científica brasileira sobre investigações polares. Códices, 10(1), 57-69. Retirado de http://132.248.9.34/hevila/ CodiceBogota/2014/vo10/no1/3.pdf

Simpson, A. (2010). The effect of Antarctic residence on energy dynamics and aerobic fitness. International Journal of Circumpolar Health, 69(3), 220-235. doi: 10.3402/ijch.v69i3. 17625

Smith, N., Kinnafick, F., \& Saunders, B. (2017). Coping strategies used during an extreme Antarctic expedition. Journal of Human Performance in Extreme Environments, 13(1). doi: 10.7771/2327-2937.1078

Steinach, M., Kohlberg, E., Maggioni, M. A., Mendt, S., Opatz, O., Stahn, A., Tiedemann, J., \& Gunga, H. C. (2015). Changes of 25-OH-Vitamin D during Overwintering at the German Antarctic Stations Neumayer II and III. PloS one, 10(12), e0144130. Retirado de https://doi.org/10.1371/journal. pone. 0144130 .

Suedfeld, P. (2012). Applying positive psychology in the study of extreme environments. Journal of Human Performance in Extreme Environments, 6(1), 21-25. Retirado de http://dx.doi. org/10.7771/2327-2937.1020

Tafforin, C. (2009). Life at the franco-italian concordia station in Antarctica for a voyage to Mars: Ethological study and anthropological perspectives. Antrocom, 5(1) 67-72. Retirado de http://www.antrocom.net/upload/sub/antrocom/050109/08Antrocom.pdf

Tothill, N. F. H., \& Martin, C. L. (2012). Winterover scientists in Antarctic Astrophysics. International Astronomical Union, 8(S288), 186-193.
Umann, J., \& Lautert, L. (2016). Resiliência, estresse, presenteísmo e capacidade para o trabalho em militares do exército. Revista de Enfermagem. UFPE online, 10(12), 4701-4704.

Vanhove, A. J., Herian, M. N., Harms, P. D., Luthans, F., \& DeSimone, J. A. (2014). Examining Psychosocial Wellbeing and performance in isolated, confined and extreme environments (Relatório Final). Retirado do sítio da NASA: http://ston.jsc.nasa.gov/collections/TRS/ techrep/TM-2015218565.pdf

Van Tilburg, M. A., Vingerhoets, A., \& Van Heck, G. (1996). Homesickness: A review of the literature. Psychological Medicine, 26(05), 899-912. doi: 10.1017/S0033291700035248

Weiss, K., Feliot, M. \& Richard, G. R. (2011). Uses of places and setting preferences in a French Antarctic station. Environment and Behavior, 2(39), 147-164. Retirado de https://halshs. archivesouvertes.fr/file/index/docid/568198/filename/ Uses_of_Places_and_Setting_Preferences_in_a_French_ Antarctic_Station.pdf

Weymouth, W., \& Steel, G. D. (2013). Sleep patterns during an Antarctic field expedition. Military Medicine, 178(4), 438-444. Retirado de http://dx.doi.org/10.7205/MILMED-D-12-00447

Zaganelli, D. M. \& Alvarez, C. E. (2011). Relationship between noise and psychological confort of the users in the Comandante Ferraz Antarctic Station. Annual Activity Report, 4, 172-177. Retirado de http://dx.doi.org/10.4322/apa.2014.084

Zimmer, M., Cabral, J. C. C. R., Borges, F. C., Coco, K. G. \& Hameister, B. R. (2013). Psychological changes arising from an Antarctic stay: Systematic overview. Estudos em Psicologia (Campinas), 30(3), 415-423. Retirado de http://dx.doi. org/10.1590/S0103-166X2013000300011 